\title{
The usefulness of point-of-care ultrasound in the atypical presentation of aortic dissection: a case series and brief review of literature
}

\author{
Gabriele Cioni \\ Emergency Department, SS Cosma and Damiano Hospital, Usl Toscana Centro, Pescia (PT), Italy
}

\begin{abstract}
Acute aortic dissection represents a surgical emergency that, in most cases, may present an atypical and non-specific clinic, making the diagnosis difficult and late. Computed tomography angiography represents the gold standard for diagnosis, but different ultrasound techniques can provide valuable additional information related to diagnosis and optimize therapy and prognostic assessment. In this case series, we describe different atypical patterns of aortic dissection associated with non-diagnostic DDimer levels. These atypical conditions escape recognized diagnostic algorithms for diagnosing aortic dissection, although the incidence of such events is far from negligible. A clinical approach in the emergency setting that provides for systematic and standardized use of bedside ultrasound could help reduce the incidence of errors and diagnostic delay, addressing the gold standard instrumental diagnostics for the reference pathology.
\end{abstract}

\section{Introduction}

Acute aortic dissection represents a surgical emergency that can be easily suspected and quickly diagnosed in the case of a typical presentation. ${ }^{1}$ In particular, lacerating and acute chest pain, anteriorly, migrating, with posterior irradiation, associated with asymmetry of the peripheral arterial wrists, represent rather suggestive findings. However, in most cases, the presentation of this severe condition is very subtle, and the symptoms are rather unspecific so as to make the diagnosis difficult and sometimes late. ${ }^{2,3}$ In confirmation of this, a high incidence of missed or incorrect diagnoses has been reported in the literature, which hangs over the correct and timely management of acute aortic syndromes. ${ }^{4,5}$ Current imaging techniques, of which computed tomography angiography (CTA) represents the gold standard, can recognize or exclude

Correspondence: Gabriele Cioni, Emergency Department, SS Cosma and Damiano Hospital, Usl Toscana Centro, Pescia (PT), Italy.

E-mail: gabrielec.83@gmail.com

Key words: Atypical aortic dissection; point-of-care ultrasound.

Received for publication: 28 September 2020.

Accepted for publication: 3 December 2020.

This work is licensed under a Creative Commons Attribution NonCommercial 4.0 License (CC BY-NC 4.0).

${ }^{\circ}$ Copyright: the Author(s), 2021

Licensee PAGEPress, Italy

Italian Journal of Medicine 2021; 15:107-110

doi:10.4081/itjm.2020.1379 alterations of the aortic wall with great efficacy; however, the patient needs an adequate selection. ${ }^{6}$

In the ADvISED Prospective Multicenter Study, a multicenter prospective observational study involving 6 hospitals in 4 countries from 2014 to 2016, the Authors assessed the diagnostic accuracy obtained by adding the D-Dimer dosage to the aortic dissection detection (ADD) risk score (ADD -RS), suggesting a low failure rate for both the ADD-RS $=0 / \mathrm{DD}-$ and ADD-RS $\leq 1 /$ DD - strategies. ${ }^{7,8}$

Different ultrasound techniques, with particular reference to transthoracic echocardiography and vascular ultrasound, and transesophageal echocardiography, intravascular ultrasound, and contrast-enhanced ultrasonography can provide valuable additional information related to diagnosis and optimize therapy and prognostic evaluation. ${ }^{9}$ In the emergency department setting, the bedside ultrasound assessment can provide a rapid clinical and diagnostic framework, particularly in the critically ill patient.

\begin{abstract}
Aim
In this case series, we describe different atypical patterns of aortic dissection associated with non-diagnostic D-Dimer levels and the role of bedside thoracic/abdominal ultrasound in suggesting this suspicion and addressing the diagnosis (Table 1).
\end{abstract}

\section{Case Reports}

The first patient, A, was an 81-year-old man who entered the emergency room for the onset of palpitations for about 4 days. Apparently they were not associated with angina or dyspnea and did not show any hemodynamic deterioration at the presentation. 
Regarding his clinical history, the patient reported essential hypertension in adequate pharmacological control with lacidipine. No other significant morbid conditions were found. Upon examination in the emergency room, the patient was alert and cooperative. We found a sinus tachycardia with an average rate of 130 bpm on the electrocardiogram and no signs of myocardial ischemia. Arterial pressure and arterial saturation in ambient air were within normal values. Hematic tests showed only a slight increase in highly sensitive troponin and values at the upper limits of the norm for pro-BNP; blood gas analysis showed a normocapnic respiratory failure, requiring low flow oxygen therapy, and a mild hyperlactacidemia.

Chest radiography showed a mild bilateral pleural effusion with increased representation of the interstitium. Despite the administration of oxygen therapy and intravenous diuretic therapy, the heart rate remained high, and hyperlactacidemia did not decrease. We performed a bedside thoracic ultrasound evaluation with finding some B lines at the bases, bilaterally, a slight pleural effusion, and, above all, a not hemodynamically significant pericardial effusion, without kinetic disturbances of cardiac walls. We extended evaluation to the abdominal aorta, discovering a diffuse atherosclerotic involvement and non-specific hemodynamic alterations on pulse wave velocity assessment. After the specialist cardiological evaluation, for the suspicion of an ascending aorta alteration ,including the valve, the patient was subjected to CT angiography of the thoracic aorta. The instrumental examination showed a type A dissection involving the aortic valve, with an indication for cardiac surgery correction. No involvement of carotid and subclavian arteries was found. D-Dimer values were slightly altered on blood tests, but not significantly if corrected for the patient's age.

The second patient, B, was a young 40-years-old man, with no significant pathologies in history who entered the DEA for pain in the right hypochondrium, suspected of biliary colic and persistence of high blood pressure, resistant to therapy with calcium antagonists. We found an isolated elevation of creatine phosphokinase at the blood tests, with a slight DDimer level elevation. There was no hyperlactacidemia or troponin alteration. Electrocardiogram was normal, with heart rate at the upper limits. At the bedside ultrasound evaluation of the abdominal aorta, we found demodulation of the hemodynamic flow, altering the vascular wall. Subsequent CT angiography of the aorta revealed a type B dissection (type III b DeBakey classification) involving the distal aorta and the right external iliac.

The third patient, C, was a young man with sudden isolated left flank pain associated with urinary retention. At the clinical evaluation, we found pain on percussion of the left side; arterial pulses were symmetrical and normal. We found marked neutrophilic leukocytosis in the blood tests, the elevation of the $\mathrm{C}$ reactive protein, and creatine phosphokinase; elevated lactacidemia was found on blood gas analysis. The patient underwent bedside ultrasound with suspicion of left renal colic; however, the ultrasound evaluation ruled out a dilation of the renal pelvis,

Table 1. Clinical characteristics and ultrasound findings of patients.

\begin{tabular}{|c|c|c|c|}
\hline & Patient A & Patient B & Patient C \\
\hline Age, y.o. & 74 & 40 & 36 \\
\hline Sex & Male & Male & Male \\
\hline Initial symptoms & Palpitations & Right hypochondrium pain & Left flank pain \\
\hline $\mathrm{SBP}, \mathrm{mmHg}$ & 120 & 160 & 150 \\
\hline DBP, $\mathrm{mmHg}$ & 80 & 100 & 90 \\
\hline Frequency rate, bpm & 130, sinus tachycardia & 100, sinus tachycardia & 90, sinus tachycardia \\
\hline D-Dimer, mcg/if EU & 750 & 470 & 510 \\
\hline $\mathrm{CPK}, \mathrm{U} / \mathrm{L}$ & 260 & 180 & 630 \\
\hline Lactate levels & 1.7 & 0.7 & 2.9 \\
\hline TT US & $\begin{array}{l}\text { Pericardial effusion, } \\
\text { basal B lines, } \\
\text { mild pleural effusion }\end{array}$ & No relevant findings & No relevant findings \\
\hline TA US & $\begin{array}{l}\text { Atheromatous abdominal aorta, } \\
\text { aspecifical hemodynamic alterations } \\
\text { on pulse wave }\end{array}$ & $\begin{array}{l}\text { Demodulation of the hemodynamic flow, } \\
\text { associated with alteration of the vascular wall } \\
\text { on the prerenal aorta }\end{array}$ & $\begin{array}{c}\text { No flow detectable } \\
\text { on the left renal artery, } \\
\text { asymmetrical flow on iliac arteries }\end{array}$ \\
\hline Type of lesion & Stanford A, DeBakey II & Stanford B, DeBakey IIIb & Stanford B, DeBakey IIIa \\
\hline
\end{tabular}


showing a hyperechogenicity of the renal cortex with almost no arterial flow detectable in the left renal artery. Moreover, the hemodynamic arterial profile was asymmetric at the level of the external iliac arteries, with a reduced systolic peak on the left side, affected by the suspected dissection.

The patient underwent CT angiography with dissection of the abdominal aorta in the prerenal tract, the complete exclusion of the left renal artery, and ongoing renal infarction.

\section{Discussion}

Aortic dissection represents a severe pathology whose treatment and prognosis depend both on the type of vascular lesion, and on the precocity of the diagnosis. ${ }^{10}$ In particular, mortality rate secondary to thoracic aortic dissection is high, and above all, time-dependent, increasing from $1 \%$ to $1.4 \%$ for each hour of treatment delay, and in any case up to $68 \%$ in the first 48 hours. ${ }^{11,12}$

The most frequent clinical presentation consists of chest pain - pulses. ${ }^{12,13}$ However, dissection can occur anywhere in the aorta, making clinical manifestation potentially unpredictable. Indeed, cases of atypical presentation are frequently described and associated with variable outcomes ${ }^{14}$ (Table 2). Acute painless aortic dissection has been described in 5\% of patients and may occur in approximately $5 \%$ of patients; in about $8 \%$ of cases, it was reported as painless and associated with syncope. Acute paraplegia has been reported rarely in $4.2 \%$ of 1805 patients with aortic dissection. ${ }^{15-18}$ Numerous other clinical reports describe a myriad of clinical presentations, potentially making the physician's job in diagnosing the disorder very difficult. ${ }^{19}$ The importance of the rapid and certain diagnosis of aortic dissection is even more true, especially when considering the differential diagnosis with acute coronary syndromes, whose therapy is potentially harmful. ${ }^{4,20}$ Literature data support the use of computed tomography for diagnosis in cases showing an indicative or suspicious clinic,${ }^{13}$ however, cases with atypical presentation (Table $2^{21-31}$ ) escape the usual diagnostic algorithms, and the suspicion is formulated only after multiple imaging studies are performed, with considerable delay.

For this reason, the clinician in the emergency department must keep the suspicion of possible dissection of the aorta high and must have access to a versatile and quick-to-use diagnostic method that allows a multisystem approach to the patient. ${ }^{21}$ In this regard, the role of point-of-care ultrasound as an ally of the emergency physician is well known. ${ }^{22}$ In this case report, the three patients, showed palpitations, pain typical of biliary colic, and renal colic, respectively. A systematic approach for managing the thorax ${ }^{23}$ and abdomen ${ }^{24}$ with bedside ultrasound method allowed the diagnosis to be delayed to a minimum.

\section{Conclusions}

Dissection of the aorta represents a hidden possibility in the shadow of multiple, apparently non-specific clinical pictures. These conditions escape recognized diagnostic algorithms for diagnosing aortic dissection, although the incidence of such events is far from negligible. A clinical approach in the emergency setting that provides for systematic and standardized use of bedside ultrasound could help reduce the incidence of errors and diagnostic delay, addressing the gold standard instrumental diagnostics for the reference pathology.

\section{References}

1. Erbel R, Aboyans V, Boileau C, et al; ESC Committee for Practice Guidelines. 2014 ESC Guidelines on the diagnosis and treatment of aortic diseases: document covering acute and chronic aortic diseases of the thoracic and abdominal aorta of the adult. The Task Force for the Diagnosis and Treatment of Aortic Diseases of the European Society of Cardiology (ESC). Eur Heart J 2014;35:2873.

Table 2. Atypical presentation of aortic dissection.

\begin{tabular}{|c|c|}
\hline Atypical presentation & Author \\
\hline Dull pain deep to the suprasternal notch & Saunders and Suzuki, $2018^{14}$ \\
\hline Pain mimicking appendicitis & Azharuddin et al., $2018^{25}$ \\
\hline Fever & Yuan, $2017^{26}$ \\
\hline Painless & Siddiqui et al., 2018:27 Ayric et al., 2006;:28 Young et al., 2002; $;{ }^{16}$ Rosen et al., 1988; ${ }^{17}$ Zull et al., $1988^{18}$ \\
\hline Neurological presentation at the onset & Bekele et al., 2017; ${ }^{29}$ Rosen et al., 1988; ${ }^{17}$ Zull et al., $1988^{18}$ \\
\hline Pleuritic chest pain & Mohamed-Yassin et al., $2019^{30}$ \\
\hline Congestive heart failure & Xiuhua et al., $2016^{31}$ \\
\hline
\end{tabular}


2. Nazerian P, Mueller C, de Matos Soeiro A, et al; ADvISED Investigators. Diagnostic accuracy of the aortic dissection detection risk score plus D-Dimer for acute aortic syndromes: The ADvISED Prospective Multicenter Study. Circulation 2018;137:250-8.

3. Klompas M. Does this patient have an acute thoracic aortic dissection? JAMA 2002;287:2262-72.

4. Hansen MS, Nogareda GJ, Hutchison SJ. Frequency of and inappropriate treatment of misdiagnosis of acute aortic dissection. Am J Cardiol 2007;99:852-6.

5. Zhan S, Hong S, Shan-Shan L, et al. Misdiagnosis of aortic dissection: experience of 361 patients. J Clin Hypertens (Greenwich) 2012;14:256-60.

6. Baliga RR, Nienaber CA, Bossone E, et al. The role of imaging in aortic dissection and related syndromes. JACC Cardiovasc Imaging 2014;7:406-24.

7. Rogers AM, Hermann LK, Booher AM, et al.; IRAD Investigators. Sensitivity of the aortic dissection detection risk score, a novel guideline-based tool for identification of acute aortic dissection at initial presentation: results from the International Registry of Acute Aortic Dissection. Circulation 2011;123:2213-8.

8. Asha SE, Miers JW. A systematic review and metaanalysis of D-dimer as a rule-out test for suspected acute aortic dissection. Ann Emerg Med 2015;66:368-78.

9. Liu F, Huang L. Usefulness of ultrasound in the management of aortic dissection. Rev Cardiovasc Med 2018;19:103-9.

10. Abarca Rozas BA, Schwarze Fieldhouse MW, Contreras Bertolo RI, et al. Atypical presentation and late diagnosis of acute aortic dissection without timely surgical treatment: case report and literature review. Medwave 2018;18:e7249.

11. Erbel R, Alfonso F, Boileau C, et al. Diagnosis and management of aortic dissection. Task Force Report. Eur Heart J 2001;22:1642-81.

12. Asouhidou I, Asteri T. Acute aortic dissection: be aware of misdiagnosis. BMC Res Notes 2009;2:25.

13. Hagan PG, Nienaber CA, Isselbacher EM, et al. The international Registry of Acute Aortic Dissection (IRAD): new insights into an old disease. JAMA 2000;16;283: 897-903.

14. Saunders T, Suzuki T. Atypical presentation of acute aortic dissection in a young competitive rower. BMJ Case Rep 2018;2018:bcr2018225712.

15. Ayrik C, Cece H, Aslan O, et al. Seeing the invisible: painless aortic dissection in the emergency setting. Emerg Med J 2006;23:24.

16. Young J, Herd AM. Painless acute aortic dissection and rupture presenting as syncope. J Emerg Med 2002; 22:171-4.

17. Rosen SA. Painless aortic dissection presenting as spinal cord ischemia. Ann Emerg Med 1988;17:840-2.

18. Zull DN, Cydulka R. Acute paraplegia: a presenting manifestation of aortic dissection. Am J Med 1988;84: 765-70.

19. Donovan EM, Seidel GK, Cohen A. Painless aortic dissection presenting as high paraplegia: a case report. Arch Phys Med Rehabil 2000;81:1436-8.

20. Kawano H, Tomichi Y, Fukae S, et al. Aortic dissection associated with acute myocardial infarction and stroke found at autopsy. Intern Med 2006;45:957-62.

21. Wroblewski R, Gibbons R, Costantino T. Point-of-care ultrasound diagnosis of an atypical acute aortic dissection. Clin Pract Cases Emerg Med 2018;2:300-3.

22. Chen L, Malek T. Point-of-care ultrasonography in emergency and critical care medicine. Crit Care Nurs Q 2018;41:94-101.

23. Lichtenstein DA. BLUE-protocol and FALLS-protocol: two applications of lung ultrasound in the critically ill. Chest 2015;147:1659-70.

24. Campbell SJ, Bechara R, Islam S. Point-of-care ultrasound in the intensive care unit. Clin Chest Med 2018;39:79-97.

25. Azharuddin M, Delacruz MA, Baughman D, Chandler P. Atypical presentation of type B aortic dissection mimicking appendicitis managed medically. BMJ Case Rep 2018;2018:bcr2018225378.

26. Yuan SM. Fever of unknown origin in aortic dissection. Z Rheumatol 2017;76:364-71.

27. Siddiqui WJ, Arif A, Khan MH, et al. An atypical case of silent aortic dissection in a peritoneal dialysis patient: a case report and review of literature. Am J Case Rep 2018;19:880-3.

28. Ayrik C, Cece H, Aslan O, et al. Seeing the invisible: painless aortic dissection in the emergency setting. Emerg Med J 2006;23:24.

29. Bekele E, Kagolanu DC, Kim M, Stephenson K. More than just muscle spasms: a rare presentation of aortic dissection. BMJ Case Rep 2017;2017:bcr2016218432.

30. Mohamed-Yassin MS, Baharudin N, Ramli AS, Hashim H. Pleuritic chest pain and fever: An unusual presentation of aortic dissection. Malays Fam Physician 2019;14:47-52.

31. Dong X, Lu J, Cheng W, Wang C. An atypical presentation of chronic Stanford type A aortic dissection during pregnancy. J Clin Anesth 2016;33:337-40. 